\title{
SOME REMARKS IN THE FOURIER ANALYSIS
}

\author{
TIKAO TATUZAWA
}

To KIYoshi Noshiro on his 60th Birthday

In this paper, $I$ should like to add some remarks to my previous note titled "Some results in the Fourier analysis" (Nagoya Math. Journal, Vol. 27, 1966). At first, we shall show that the orthnormal set $\exp \left[2 \pi i\left(m_{1} x_{1}+\cdots+m_{n} x_{n}\right)\right]$ is complete in the Hilbert space $L^{2}$ over the unit cube $E=\left\{\left(x_{1}, \ldots, x_{n}\right)\right.$; $\left.0 \leqq x_{j} \leqq 1(1 \leqq j \leqq n)\right\}$, where the inner product $\langle f, g\rangle$ for $f, g \in L^{2}$ over $E$ is defined by

$$
\int \cdots \cdot \int f\left(x_{1}, \ldots, x_{n}\right) \bar{g}\left(x_{1}, \ldots, x_{n}\right) d x_{1} \cdots d x_{n}
$$

This means that if $f \in L^{2}$ over $E$ and

$$
\left\langle f, \exp \left[2 \pi i\left(m_{1} x_{1}+\cdots+m_{n} x_{n}\right)\right]\right\rangle=0
$$

for any integral values $m_{1}, \ldots, m_{n}$, then $f=0$ almost everywhere. To prove this, we define the set function

$$
F(S)=\int \cdots \int f\left(u_{1}, \ldots, u_{n}\right) d u_{1} \cdots d u_{n}
$$

If $S$ is any interval in $E$, then clearly $F(S)=0$, in virtue of Lemma 2 in the paper cited above. Hence, if $S$ is a closed set in $E$, accordingly if $S$ is a measurable set in $E$, then $F(S)=0$. Let $E_{n}=\left\{\left(x_{1}, \ldots, x_{n}\right) ;\left(x_{1}, \ldots, x_{n}\right) \in E, f\left(x_{1}\right.\right.$, $\left.\left.\ldots, x_{n}\right) \geqq \frac{1}{n}\right\}$ and $E_{n}^{\prime}=\left\{\left(x_{1}, \ldots, x_{n}\right) ;\left(x_{1}, \ldots, x_{n}\right) \in E, f\left(x_{1}, \ldots, x_{n}\right) \leqq-\frac{1}{n}\right\}$. Since $0=F\left(E_{n}\right) \geqq \frac{1}{n} m\left(E_{n}\right)$ and $0=F\left(E_{n}^{\prime}\right) \leqq-\frac{1}{n} m\left(E_{n}^{\prime}\right)$, we obtain $m\left(E_{n}\right)=$ $m\left(E_{n}^{\prime}\right)=0$. From this consideration, we have $m\left\{\left(x_{1}, \ldots, x_{n}\right) ;\left(x_{1}, \ldots, x_{n}\right) \in E\right.$, $\left.f\left(x_{1}, \ldots, x_{n}\right) \neq 0\right\}=0$. We get, therefore, in the usual way, the following

TheOREM 3. Let $f\left(x_{1}, \ldots, x_{n}\right), g\left(x_{1}, \ldots, x_{n}\right)$ be $L^{2}$-integrable in the unit cube $E$ and set $a\left(m_{1}, \ldots, m_{n}\right)=\left\langle f, \exp \left[2 \pi i\left(m_{1} x_{1}+\cdots+m_{n} x_{n}\right)\right]\right\rangle, b\left(m_{1}, \ldots\right.$,

Receved June 29, 1966. 
$\left.m_{n}\right)=\left\langle g, \exp \left[2 \pi i\left(m_{1} x_{1}+\cdots+m_{n} x_{n}\right)\right]\right\rangle$. Then we have

$$
\begin{aligned}
& \int \ldots \int f\left(x_{1}, \ldots, x_{n}\right) \bar{g}\left(x_{1}, \ldots, x_{n}\right) d x_{1} \cdots d x_{n}= \\
& \qquad \sum_{m_{1} \ldots m_{n}=-\infty} \ldots \sum^{\infty} a\left(m_{1}, \ldots, m_{n}\right) \bar{b}\left(m_{1}, \ldots, m_{n}\right) .
\end{aligned}
$$

Next, we note that if

$$
\frac{\partial^{p_{1}+\cdots+p_{n}}}{\partial^{p_{1}} x_{1} \cdots \partial^{p_{n}} x_{n}} f\left(x_{1}, \ldots, x_{n}\right) \quad\left(0 \leqq p_{j} \leqq 2\right)
$$

are continuous and $L$-integrable over $X$, the whole $n$-dimensional Euclidean space, then

$$
g\left(v_{1}, \ldots, v_{n}\right)=\int \ldots \int_{x}^{2 \pi i\left(v_{1} u_{1}+\cdots+v_{n} u_{n}\right)} f\left(u_{1}, \ldots, u_{n}\right) d u_{1} \cdots d u_{n}
$$

is $L$-integrable over $X$. Consequently, by Theorem 2 of the paper cited above, the Fourier-transform formula

$$
f\left(x_{1}, \ldots, x_{n}\right)=\int \cdots \int_{x} e^{-2 \pi i\left(v_{1} x_{1}+\cdots+v_{n} x_{n}\right)} g\left(v_{1}, \ldots, v_{n}\right) d v_{1} \cdots d v_{n}
$$

holds.

Proof. We take $a_{m}$ such that

$$
a_{1}<a_{2}<\cdots<a_{m} \rightarrow \infty \quad(\text { as } m \rightarrow \infty),
$$

and define

$$
\begin{aligned}
& \left.l_{m}(t)=a_{m}^{2}-t\right)^{2 n}\left(t-\frac{a_{m}^{2}}{2}\right)^{2 n} \\
& k_{m}(r)={ }_{-}^{1} \int_{a_{m}^{2} / 2}^{r} l_{m}(t) d t, \text { where } C=\int_{a^{2} m / 2}^{a^{2} m} l_{m}(t) d t, \\
& h_{m}\left(x_{1}, \ldots, x_{n}\right)=\left\{\begin{array}{cc}
1 & x_{1}^{2}+\cdots+x_{n}^{2} \leqq \frac{a_{m}^{2}}{2} \\
1-k_{m}\left(x_{1}^{2}+\cdots+x_{n}^{2}\right) & \frac{a_{m}^{2}}{2} \leqq x_{1}^{2}+\cdots+x_{n}^{2} \leqq a_{m}^{2} \\
0 & a_{m}^{2} \leqq x_{1}^{2}+\cdots+x_{n}^{2},
\end{array}\right.
\end{aligned}
$$

and set

$$
f_{m}\left(x_{1}, \ldots, x_{n}\right)=f\left(x_{1}, \ldots, x_{n}\right) h_{m}\left(x_{1}, \ldots, x_{n}\right) .
$$

By partial integration, 


$$
\begin{aligned}
& g_{m}\left(v_{1}, \ldots, v_{n}\right)=\int_{X} \int e^{2 \pi i\left(v_{1} u_{1}+\cdots+v_{n} u_{n}\right)} f_{m}\left(u_{1}, \ldots, u_{n}\right) d u_{1} \cdots d u_{n} \\
&= \int_{-a_{m}}^{a_{n i}} \ldots \int_{-a_{m}}^{a_{m}}\left\{\left[\frac{\exp \left[2 \pi i\left(v_{1} u_{1}+\cdots+v_{n} u_{n}\right)\right]}{2 \pi i v_{1}} f_{m}\left(u_{1}, \ldots, u_{n}\right)\right]_{-a_{m}}^{a_{m}}\right. \\
&\left.\quad-\int_{-a_{m}}^{a_{m}} \frac{\exp \left[2 \pi i\left(v_{1} u_{1}+\cdots+v_{n} u_{n}\right)\right]}{2 \pi i v_{1}} \frac{\partial}{\partial u_{1}} f_{m}\left(u_{1}, \ldots, u_{n}\right)\right\} d u_{2} \cdots d u_{n} \\
&= \cdots \\
& \frac{(-1)^{q_{2}+\cdots+a_{n}}}{(2 \pi i)^{q_{1}+\cdots+a_{n}}} \int_{-a_{m}}^{a_{m}} \cdots \int_{-a_{m}}^{a_{m}} \frac{\exp \left[2 \pi i\left(v_{1} u_{1}+\cdots+v_{n} u_{n}\right)\right]}{v_{1}^{q_{1}} \cdots v_{n}^{q_{n}}} \cdot \\
& \frac{\partial^{q_{1}+\cdots+a_{n}}}{\partial u_{1}^{q_{1}} \cdots \cdot \partial u_{n}^{q_{n}}} f_{m}\left(u_{1}, \ldots, u_{n}\right) d u_{1} \cdots d u_{n},
\end{aligned}
$$

where $q_{j}$ are taken such that $q_{j}=2$ if $\left|v_{j}\right| \geqq 1$ and $q_{j}=0$ if $\left|v_{j}\right|<1$. Since

$$
\frac{\partial^{p_{1}+\cdots+p_{n}}}{\partial x_{1}^{p_{1}} \cdots \cdot \partial x_{n}^{p_{n}}} h_{m}\left(x_{1}, \ldots, x_{n}\right)
$$

are uniformly bounded for all $m$, we obtain

$$
g_{m}\left(v_{1}, \ldots, v_{n}\right)=0\left(\frac{1}{\left|v_{1}\right|^{q_{1}} \cdots\left|v_{n}\right|^{q_{n}}}\right)
$$

By the Lebesgue dominated convergence theorem, sending $m \rightarrow \infty$,

$$
g\left(v_{1}, \ldots, v_{n}\right)=0\left\{\operatorname{Min}\left(1, \frac{1}{v_{1}^{2}}\right) \cdots \operatorname{Min}\left(1, \frac{1}{v_{n}^{2}}\right)\right\}
$$

from which we can infer that $g\left(v_{1}, \ldots, v_{n}\right)$ is $L$-integrable over $X$.

Finally I should like to express my thanks to the authors who presented the above problems, especially to Bochner, Hecke, Siegel, Takagi and Weyl.

\section{Institute of Mathematics}

College of General Education

University of Tokyo. 